\title{
Isolated Cardiac Sarcoidosis Mimicking Arrhythmogenic Right Ventricular Cardiomyopathy
}

\author{
Hirotaka Waki ${ }^{1}$, Kazuo Eguchi ${ }^{1}$, Shinichi Toriumi ${ }^{1}$, Tomokazu Ikemoto ${ }^{1}$, \\ Tsukasa Suzuki ${ }^{2}$, Noriyoshi Fukushima ${ }^{2}$ and Kazuomi Kario ${ }^{1}$
}

\begin{abstract}
:
The diagnosis of cardiac sarcoidosis (CS) has become easier due to advances in imaging modalities, but we sometimes encounter difficult-to-diagnose patients. We herein report the case of a 60-year-old Japanese woman who was diagnosed with isolated CS, although she also met the diagnostic criteria of arrhythmogenic right ventricular cardiomyopathy (ARVC). A histological examination by an endomyocardial biopsy of the right ventricle revealed the typical findings of granulomatous change for CS. Although she did not show any characteristics of systemic sarcoidosis, oral prednisolone treatment was introduced, and she achieved a good response. This case shows that the characteristics of CS can overlap with the diagnostic criteria of ARVC, and that a histological examination is essential for the correct diagnosis of CS.
\end{abstract}

Key words: cardiac sarcoidosis, arrhythmogenic right ventricular cardiomyopathy, endomyocardial biopsy, epsilon waves

(Intern Med 57: 835-839, 2018)

(DOI: 10.2169/internalmedicine.9395-17)

\section{Introduction}

Cardiac sarcoidosis (CS) is seen in 5\% of systemic sarcoidosis cases and is categorized as infiltrative cardiomyopathy. Sarcoidosis can involve any location of the heart, and the morphological features of CS vary: regional wall motion abnormality, aneurysm, septal wall thinning, dilated left ventricle, and an impaired right or left ventricular systolic or diastolic function (1). A clinical diagnosis of CS is sometimes not definitive, especially in cases of "isolated cardiac sarcoidosis" (2).

Arrhythmogenic right ventricular cardiomyopathy (ARVC) is characterized by pathological fibrofatty replacement from the epicardium toward the endocardium that leads to right ventricular dysfunction (3). The advanced stage of ARVC can involve the left ventricle, and the cardiac features of advanced ARVC mimic those of CS. Both CS and ARVC can present as biventricular heart failure and carry risks of lifethreatening ventricular arrhythmias or sudden cardiac death (4). CS is an indication for early treatment with corti- costeroids or other immunosuppressive agents. In contrast, there are many treatments for ARVC to reduce the risk of developing symptoms or sudden cardiac death, but there is no cure for ARVC at present. Because the treatment strategies for CS and ARVC are very different, an accurate diagnosis is mandatory.

Some case studies have shown that CS may overlap with ARVC in clinical presentations (5-7). Therefore, a careful diagnostic process is needed for the initial diagnoses of CS and ARVC in clinical practice. In the case described below, we treated a patient with CS whose clinical diagnosis was very similar to ARVC, but histological confirmation with a right ventricular (RV) endomyocardial biopsy led to the correct diagnosis of CS.

\section{Case Report}

A permanent pacemaker was implanted in a 60 -year-old Japanese woman due to complete atrioventricular block (CAVB) at a nearby hospital 1 year prior to her admission to our institution. At that time, the left ventricular (LV) con-

${ }^{1}$ Division of Cardiovascular Medicine, Department of Medicine, Jichi Medical University School of Medicine, Japan and ${ }^{2}$ Department of Diagnostic Pathology, Jichi Medical University Hospital, Japan

Received: April 25, 2017; Accepted: July 27, 2017; Advance Publication by J-STAGE: December 8, 2017

Correspondence to Dr. Kazuo Eguchi, ke112@jichi.ac.jp 


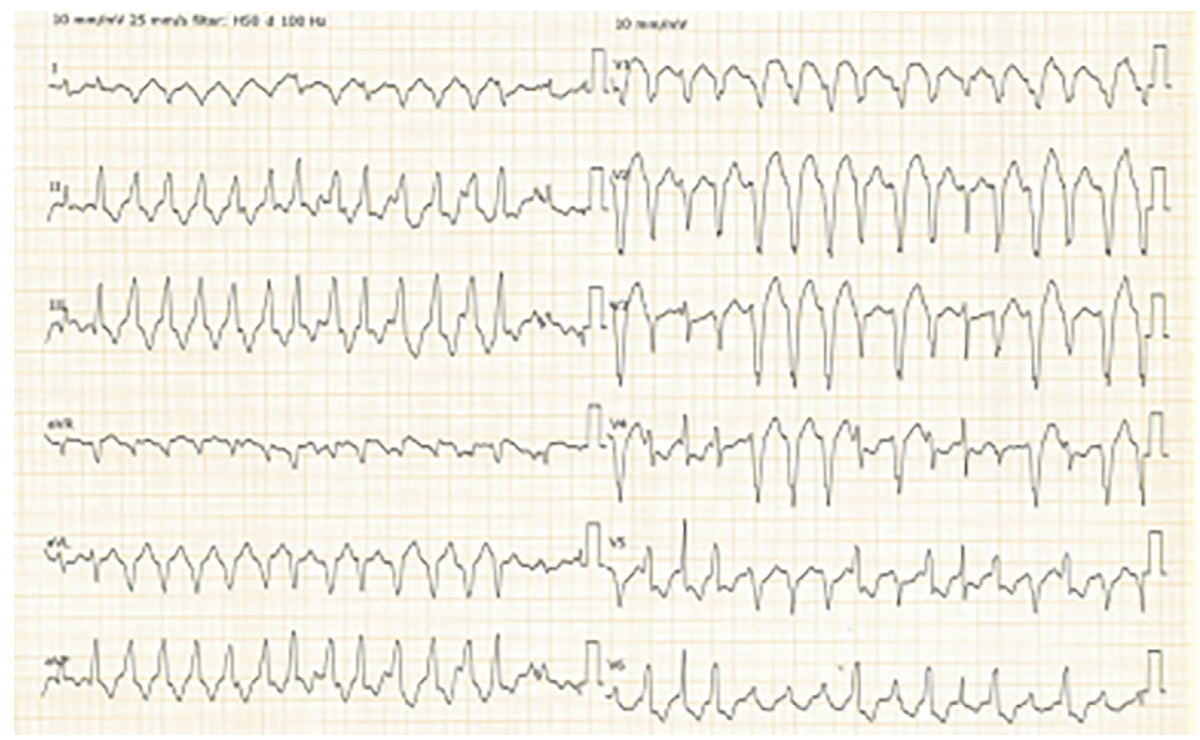

Figure 1. The patient's ECG on admission showed sustained ventricular tachycardia of left branch block morphology at the inferior axis.

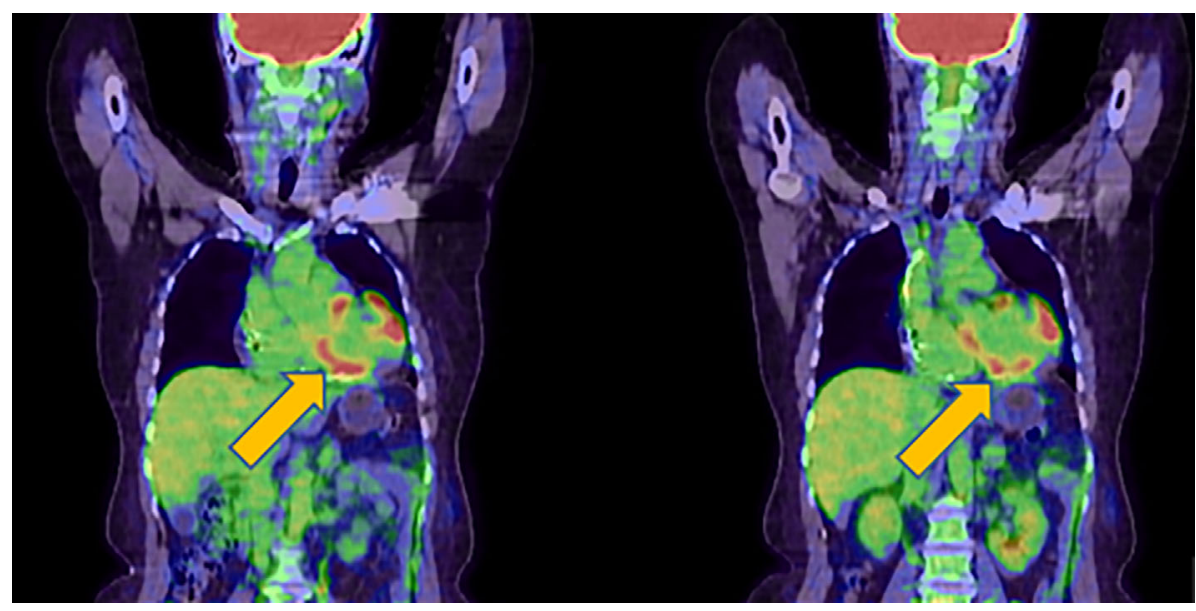

Figure 2. Serial ${ }^{18}$ F-FDG PET images of the patient, a 60-year-old woman. The images demonstrated some focal uptake in the septal and inferior walls of the left ventricle but not in the right ventricle. ${ }^{18}$ F-FDG PET: ${ }^{18}$ F-fluorodeoxyglucose positron emission tomography

traction by LV ejection fraction (LVEF) was 60\%. She had no known history or family history of heart diseases.

However, two months after the pacemaker implantation, the patient began to feel palpitations. Twenty-four-hour Holter electrocardiogram (ECG) monitoring was performed, and premature ventricular contractions (PVCs) and non-sustained ventricular tachycardia were identified when the patient felt a palpitation (PVCs/total beats $=7,355 / 97,689$, longest PVC: 5 beats). Ten months after the pacemaker implantation, the patient began to feel external dyspnea, and her cardiac function was shown by transthoracic echocardiography (TTE) to be reduced (LVEF 40\%).

At 11 months after the pacemaker implantation, the patient was hospitalized due to heart failure with sustained ventricular tachycardia (VT) (Fig. 1). The patient's heart failure was treated by guideline-based standard treatment, and amiodarone (loading dose $400 \mathrm{mg} / \mathrm{day}$, maintenance dose $200 \mathrm{mg}$ /day) was started for her sustained VT; her general condition subsequently improved. The underlying cause of her heart failure was investigated. TTE showed a reduced LVEF (31\%), reduced right ventricular function (fractional area change $27 \%$ ), and dilated left and right ventricles, but regional wall thinning was not observed. On ${ }^{67} \mathrm{Ga}$ scintigraphy, the non-specific uptake of Gallium was observed in the myocardium. ${ }^{18} \mathrm{~F}$-fluorodeoxyglucose positron emission tomography $\left({ }^{18} \mathrm{~F}\right.$-FDG PET) images demonstrated some focal uptake in the septal and inferior wall of the left ventricle, but not in the right ventricle (Fig. 2). The maximum standardized uptake value (SUVmax) was 7.5 in the LV septal wall, 7.0 in the LV lateral wall, and 6.9 in the LV inferior wall.

Based on the latest version of the Guidelines for Diagno- 


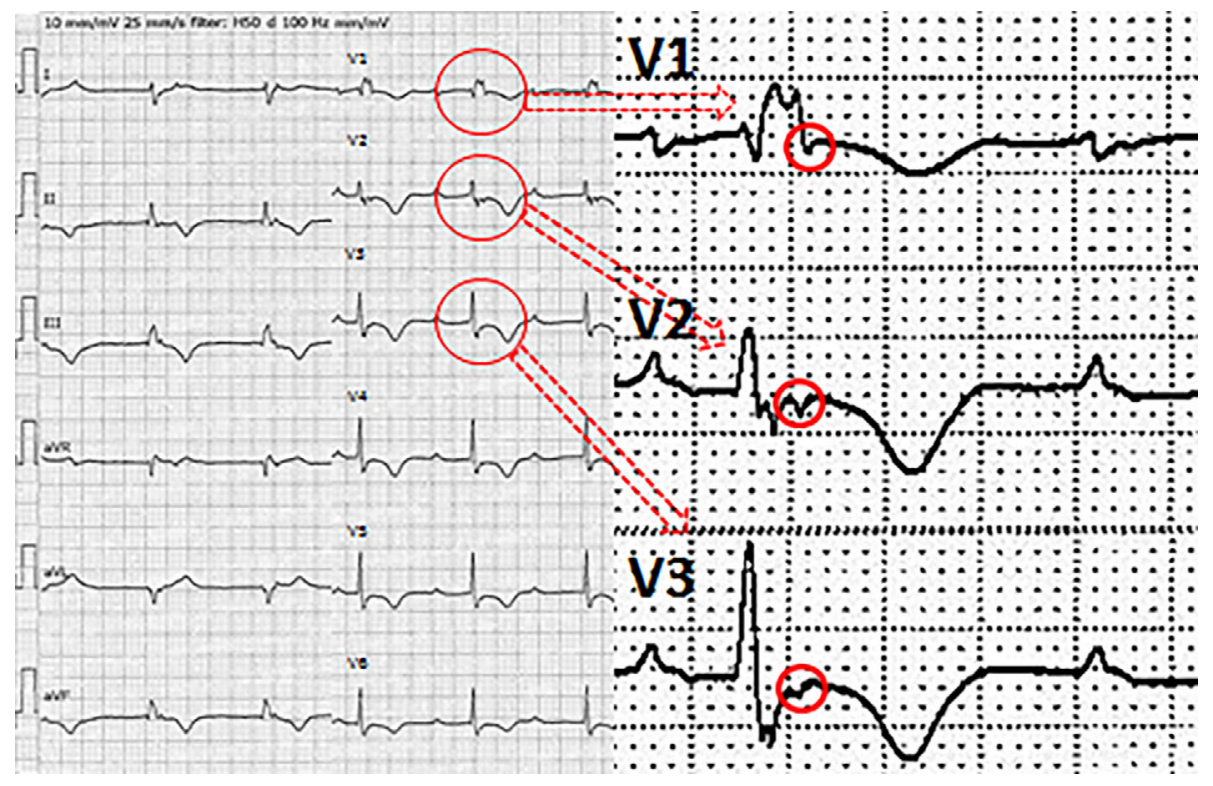

Figure 3. The patient's ECG showed small deflections at the end of the complex in V1-3 that were considered epsilon waves. ECG: electrocardiogram

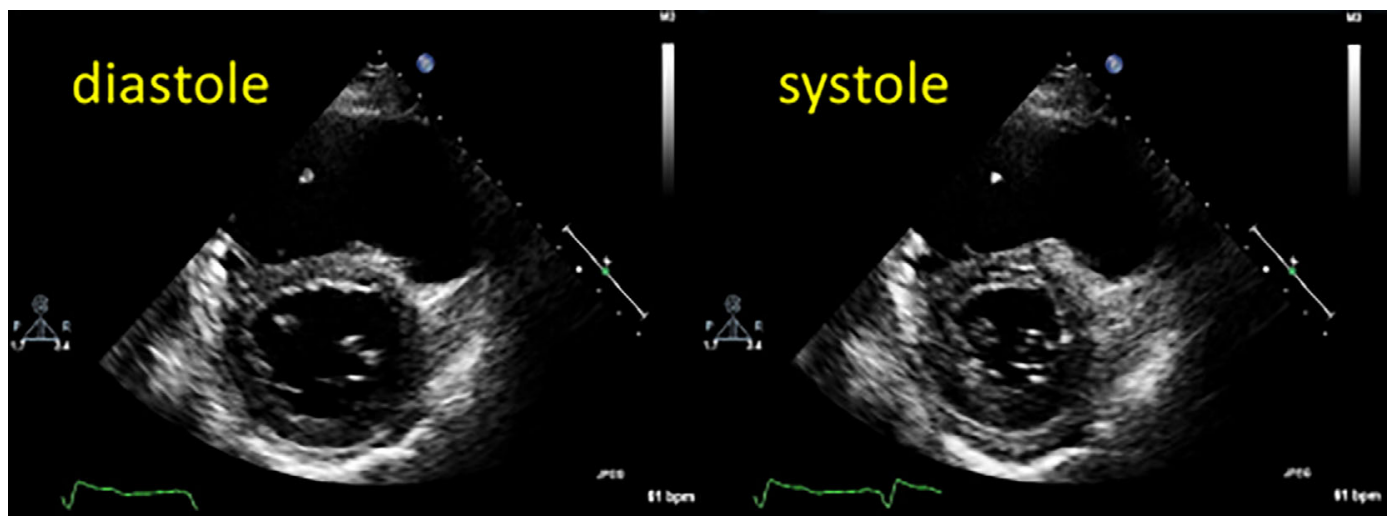

Figure 4. TTE showed a reduced LVEF (31\%), reduced right ventricular function (fractional area change $27 \%$ ), and dilated left and right ventricles. The thickness of the basal interventricular septum was normal. LVEF: left ventricular ejection fraction, TTE: transthoracic echocardiography

sis and Treatment of Cardiac Sarcoidosis (Japanese Circulation Society 2016) (8), this patient did not meet the clinical criteria of systemic sarcoidosis: blood tests, chest X-ray, and chest computed tomography did not show any evidence of sarcoidosis, and ophthalmologists did not find any signs of sarcoidosis by standard methods. However, the patient met three major criteria (CAVB and sustained VT, LV dysfunction, and ${ }^{18} \mathrm{~F}-\mathrm{FDG}$ uptake) and one minor criterion (nonsustained VT and PVCs) for CS.

However, ECG of her own beats showed small deflections at the end of the QRS complex in V1-3 that was suspected to be epsilon waves (Fig. 3). TTE and right ventriculography showed a dilated and impaired wall motion of right ventricle (Fig. 4), and >1,000 PVCs were recorded by 24hour Holter ECG. Given these collective findings, the patient met two major criteria (global RV dysfunction, and inverted T-waves in right precordial leads) and two minor criteria [sustained ventricular tachycardia of left bundle branch block (LBBB) morphology at the inferior axis, and nonsustained VT of unknown axis $>500$ PVCs per 24 hours] of an international Task Force for the diagnosis of ARVC (4), as described in the Discussion section below. At this point in the patient's course, we thus considered the possibility of ARVC rather than CS. In addition, pacing-induced cardiomyopathy was also a possible cause of her condition, as her pacemaker was VDD (the pacemaker senses atrial and ventricular events but can only pace the ventricle). With clinical differential diagnoses of CS, ARVC, or pacing-induced cardiomyopathy, we performed a right ventricular endomyocardial biopsy to achieve a histological and definitive diagnosis.

However, the first pathological report revealed only slight lymphocytic infiltration in the myocardial tissues, which was diagnosed as a non-specific finding histologically. We reevaluated the biopsy specimen by deep cutting and observed granulomatous lesions in the biopsied specimens; such lesions are not a typical pathology in CS, but they do indicate 


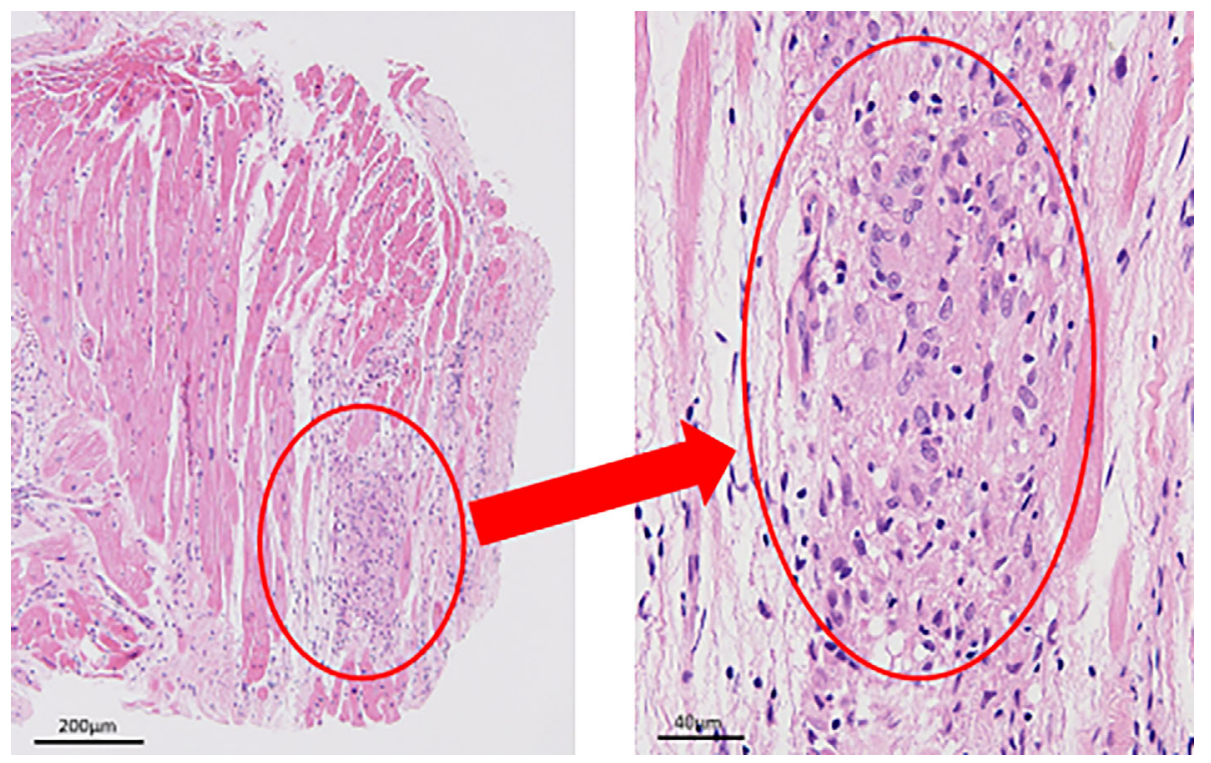

Figure 5. A sample of endomyocardial tissue from the right ventricular myocardial biopsy, obtained by deep cutting. The biopsy showed the accumulation of epithelioid cells and multinucleated giant cells. Granuloma was observed, and we were thus able to make the diagnosis of cardiac sarcoidosis (CS).

CS (Fig. 5). Thus, the patient's diagnosis was confirmed as $\mathrm{CS}$, and we initiated oral prednisolone treatment $(30 \mathrm{mg} /$ day). She achieved a good response and has been well for about six months since the prednisolone treatment.

\section{Discussion}

We experienced a patient who met several of the international Task Force diagnostic criteria for ARVC but was ultimately diagnosed with isolated CS based on a myocardial biopsy. This Task Force for the diagnosis of ARVC (3) describes six categories: global or regional dysfunction and structural alterations, tissue characterization, repolarization abnormalities, depolarization and conduction abnormalities, arrhythmias, and family history. The diagnosis of ARVC requires meeting two major criteria, one major and two minor criteria, or four minor criteria. Our patient met two major criteria (global RV dysfunction, and inverted T-waves in right precordial leads) and two minor criteria [sustained ventricular tachycardia of left bundle branch block (LBBB) morphology at the inferior axis, and non-sustained VT of unknown axis >500 PVCs per 24 hours]. In addition, our patient also met the criteria provided by an earlier (1994) international Task Force for the diagnosis of ARVC with a lower sensitivity but higher specificity than the 2010 diagnostic criteria (9).

It has been reported that some patients with CS can meet the diagnostic criteria for ARVC (5-7). One reason for this is because CS can cause RV involvement, ventricular tachycardia, or delayed activation of myocardium, which are commonly seen in patients with ARVC. These characteristics of the myocardium $(3,10)$ can be seen in both fibro-fatty replacements in ARVC and granulomatous infiltration in CS.
In a study that examined the differences between CS and ARVC, RV involvement was demonstrated in $48 \%$ of the 40 patients with CS and all 21 of the patients with ARVC, and LV involvement was demonstrated in $65 \%$ of the CS patients and only in $5 \%$ of the ARVC patients (11). As mentioned above, RV dysfunction often occurs in CS but is not a very good indicator for the diagnosis of CS, whereas LV dysfunction ( $\mathrm{LVEF}<50 \%$ ) is more likely to occur in CS than in ARVC (12). In the present patient's case, even though the diagnostic criteria of ARVC were sufficiently met, CS was more likely than ARVC because the left ventricle was involved.

Our patient showed epsilon waves, which was seen after the QRS complex at the beginning of the ST segment. Epsilon waves have been reported to be found in 30-33\% of patients with ARVC and are regarded as a reflection on the ECG presentation of delayed activation of some right ventricular fibers $(13,14)$. In the present case, a notch was observed just at the point when we recorded her own beats, so we considered the notch to be the epsilon waves. In a study of 49 patients who met the criteria of ARVC, the patients with epsilon waves were significantly more likely to have diffuse RV involvement than those without the waves $(86 \%$ vs. $62 \%, \mathrm{p}=0.002)(15)$. Conversely, epsilon waves were present in $73 \%$ of patients with diffuse RV involvement but only in $25 \%$ of those with localized disease $(p=0.002)$. Those findings indicate that the presence of epsilon wave is an indicator of diffuse RV involvement of ARVC. However, ECG changes associated with ARVC, such as T-wave inversion at leads V1-V4 and epsilon waves, are sometimes also observed in CS patients (5). We should acknowledge that epsilon waves can be seen in any condition that induces a conduction delay of the RV wall. 
The multifocal ${ }^{18}$ F-FDG PET uptake was observed in the LV wall, but no uptake was observed in the RV in our patient. We propose two possible explanations for this lack of an ${ }^{18}$ F-FDG uptake in the RV. First, the RV was already dilated and showed remodeling, and active inflammation was not present at the time of the diagnosis. Second, the RV myocardium is thinner than that in the $\mathrm{LV}$ and not likely to have lesions large enough to have any ${ }^{18} \mathrm{~F}$-FDG uptake, because of the influence of the partial volume effect on PET activity. ${ }^{18}$ F-FDG PET is a useful method of diagnosing CS, but it is not used to diagnose ARVC (10). The ${ }^{18} \mathrm{~F}$-FDG PET findings that are specific to ARVC are limited, as ARVC is not typically related to metabolic activity, which can increase the ${ }^{18} \mathrm{~F}$-FDG uptake. However, inflammatory changes have been reported in ARVC patients, and these changes can increase the RV uptake of ${ }^{67} \mathrm{Ga}$ (16).

The 2010 international Task Force for the diagnosis of ARVC (9) suggests the use of an endomyocardial biopsy from right ventricular free wall (RVFL) to diagnose ARVC, but a biopsy from the RVFL is somewhat risky and controversial. An endomyocardial biopsy has high specificity but low sensitivity for the diagnosis of ARVC (specificity 92\%, sensitivity 67\%) or CS (specificity 99-100\%, sensitivity $30 \%$ ), and therefore an endomyocardial biopsy may not always be performed in these cases $(17,18)$. However, it was reported that, among patients with suspected ARVC, the incidence of CS was very high $(15 \%)$ when it was evaluated by a standard protocol including an endomyocardial biopsy (12). Therefore, as shown in this patient, who is suspected of having isolated CS, an endomyocardial biopsy is very important for the correct diagnosis and treatment of CS (8).

In conclusion, the present case confirms that clinical characteristics of CS can overlap with the diagnostic criteria of ARVC, and a histological examination is essential for the correct diagnosis of CS.

The authors state that they have no Conflict of Interest (COI).

\section{References}

1. Burstow DJ, Tajik AJ, Bailey KR, DeRemee RA, Taliercio CP. Two-dimensional echocardiographic findings in systemic sarcoidosis. Am J Cardiol 63: 478-482, 1989.

2. Okada DR, Bravo PE, Vita T, et al. Isolated cardiac sarcoidosis: a focused review of an under-recognized entity. J Nucl Cardiol 2016 [Epub ahead of print] Erratum in J Nucl Cardiol. 2016.

3. Corrado D, Link MS, Calkins H. Arrhythmogenic right ventricular cardiomyopathy. N Engl J Med 376: 61-72, 2017.
4. Romero J, Mejia-Lopez E, Manrique C, Lucariello R. Arrhythmogenic right ventricular cardiomyopathy (ARVC/D): a systematic literature review. Clin Med Insights Cardiol 7: 97-114, 2013.

5. Philips B, Madhavan S, James CA, et al. Arrhythmogenic right ventricular dysplasia/cardiomyopathy and cardiac sarcoidosis distinguishing features when the diagnosis is unclear. Circ Arrhythm Electrophysiol 7: 230-236, 2014.

6. Terasaki F, Ishizaka N. Cardiac sarcoidosis and arrhythmogenic right ventricular cardiomyopathy-potential differential diagnosis for arrhythmogenic ventricular cardiomyopathy. Intern Med 55: 1041-1042, 2016.

7. Vasatruro S, Ploeg DE, Buitrago G, et al. Right ventricular cardiomyopathy meeting the arrhythmogenic right ventricular dysplasia revised criteria? Don't forget sarcoidosis! Korean J Radiol 16: 668-672, 2015.

8. Guidelines for Diagnosis and Treatment of Cardiac Sarcoidosis (The Japanese Circulation Society 2016).

9. Marcus FI, McKenna WJ, Sherrill D, et al. Diagnosis of arrhythmogenic right ventricular cardiomyopathy/dysplasia: proposed modification of the Task Force Criteria. Eur Heart J 31: 806-814, 2010.

10. Hulten E, Aslam S, Osborne M, et al. Cardiac sarcoidosis-state of the art review. Cardiovasc Diagn Ther 6: 50-63, 2016.

11. Steckman DA, Schneider PM, Schuller JL, et al. Utility of cardiac magnetic resonance imaging to differentiate cardiac sarcoidosis from arrhythmogenic right ventricular cardiomyopathy. Am J Cardiol 110: 575-579, 2012.

12. Vasaiwala SC, Finn C, Delpriore J, et al. Prospective study of cardiac sarcoid mimicking arrhythmogenic right ventricular dysplasia. J Cardiovasc Electrophysiol 20: 4736, 2009.

13. Nasir K, Bomma C, Tandri H, et al. Electrocardiographic features of arrhythmogenic right ventricular dysplasia/cardiomyopathy according to disease severity: a need to broaden diagnostic criteria. Circulation 110: 1527-1534, 2004.

14. Fontaine G, Guiraudon G, Frank R. Stimulation studies and epicardial mapping in ventricular tachycardia: study of mechanisms and selection for surgery. In: Reentrant Arrhythmias. Kulbertus HE, Ed. MTP Publishers, Lancaster, 1977: 334-384.

15. Wu S, Wang P, Hou Y, et al. Epsilon wave in arrhythmogenic right ventricular dysplasia/cardiomyopathy. PACE 32: 59-63, 2009.

16. Campian M, Ververne H, Hardziyenka M, et al. Assessment of inflammation in patients with arrhythmogenic right ventricular cardiomyopathy/dysplasia. Eur J Nucl Med Mol Imaging 37: 20792085, 2010.

17. Aredehali H, Howard DL, Hariri A, et al. A positive endomyocardial biopsy result for sarcoid is associated with poor prognosis in patients with initially unexplained cardiomyopathy. Am Heart $\mathbf{J}$ 150: 459-463, 2005.

18. Quata G, Elliott PM. Diagnostic criteria for arrhythmogenic right ventricular cardiomyopathy. Rev Esp Cardiol (Engl Ed) 65: 599605, 2012.

The Internal Medicine is an Open Access article distributed under the Creative Commons Attribution-NonCommercial-NoDerivatives 4.0 International License. To view the details of this license, please visit (https://creativecommons.org/licenses/ by-nc-nd/4.0/).

(C) 2018 The Japanese Society of Internal Medicine Intern Med 57: 835-839, 2018 